\title{
Wind Accretion by Compact Objects: The "Flip-Flop" Instability
}

\author{
Mario Livio \\ Space Telescope Science Institute \\ 3700 San Martin Drive \\ Baltimore, MD 21218 \\ and \\ Dept. of Physics, Technion \\ Haifa 32000 \\ Israel
}

\begin{abstract}
The problem of the stability of wind accretion onto compact objects is examined. Recent analytical and numerical calculations show that in two dimensions, Bondi-Hoyle accretion flows are unstable to a "flip-flop" instability. The instability can manifest itself as bursts in the accretion rate and as a random walk-type spin-up, spin-down behaviour of the accreting compact object. The nature of the flow in three dimensions needs further clarification. Possible observational implications are reviewed.
\end{abstract}

\section{Introduction}

The problem of accretion from a stellar wind onto compact objects is important in a variety of astrophysical configurations. For example: (1) massive X-ray binaries, in which neutron stars or black holes accrete from the wind of OB companions, (2) symbiotic binaries and barium stars, in which white dwarfs accrete from the winds of cool giants and (3) $\zeta$ Aur binaries, in which main sequence stars accrete from the winds of cool companions. Some aspects of the problem were discussed in reviews by Lamb (1988), Nagase (1989) and Livio (1991). Here I wish to discuss mainly the question of the stability of the flow.

The first suggestion that accretion from stellar winds may be unstable, came from attempts to determine the rate of accretion of angular momentum from a medium containing a density or velocity gradient (Davies and Pringle 1980, Livio 1988). High resolution, two dimensional (2D) numerical calculations of accretion flows with such gradients, showed that the flow did not reach a steady state in some cases. Instead, the shock-cone oscillated from side-to-side in a "flip-flop" motion, accompanied by brief periods of disk formation (Matsuda, Inoue and Sawada 1987, Taam and Fryxell 1988, Fryxell and Taam 1988, Sawada et al. 1989). In an attempt to determine the reality of this phenomenon, as well as its physical nature, a group of us embarked on an extensive numerical (Matsuda et al. 1991a, 1991b) and analytical (Soker 1990, 1991, Livio et al 1991) effort. Two-dimensional calculations which included radiative effects were recently performed by Taam, Fu and Fryxell (1991, see also Blondin et al 1991). In Section II, I shall describe the results of some of these works. Some possible observational implications are discussed in Section III and remaining problems are described in Section IV. 


\section{The Stability of Wind Accretion}

The two main questions that emerged from the 2D calculations of Matsuda et al (1987) and Taam and Fryxell (1988) were the following: (1) Does the development of the flip-flop instabitity (in 2D) require the presence of a density or velocity gradient or is the instability an intrinsic property of Bondi-Hoyle (1955) accretion flows (at least in two-dimensions)?

(2) Is the instability an artifact of the use of two-dimensions (in the numerical calculations) to describe a flow which is really three-dimensional, or does the instability appear also in three-dimensional (3D) calculations? (in which case it is more likely that it represents a genuine property of realistic flows).

In order to answer the first question, Matsuda et al (1991a) performed a series of calculations in $2 \mathrm{D}$, in which the medium was taken to be homogeneous at infinity. Their results can be summarized as follows:

(1) In all the cases in which the size of the accreting object was taken to be sufficiently small $\left(R_{a c c} / 16\right.$, where $R_{a c c}$ is the accretion radius) and for a specific heats ratio $\gamma \geq 4 / 3$, the flip-flop instability appeared and eventually took the form of a violent, aperiodic oscillation. In all of these cases, the violent oscillation was preceded by the formation of a wide, domeshaped shock, on the upstream side of the accreting object (Figs. 1 and 2).

(2) In nearly isothermal calculations $(\gamma=1.1,1.05,1.005)$, the flip-flop oscillation was quite smooth and quasi-periodic (Fig. 3). In a calculation in which the size of the accreting object was suddenly increased (from $R_{a c c} / 16$ to $5 R_{a c c} / 16$ ), after the increase, the oscillation also became quasi-periodic (Fig. 4)). All of these flows were characterized by relatively narrow shock-cones.

(3) When the size of the accreting object was taken to be large $\left(5 R_{a c c} / 16\right)$ throughout the run, the flow was found to be stable for the duration of the run $(t=45$, in units of $\mathrm{R}_{a c c} / C_{s}$ where $C_{s}$ is the speed of sound at infinity). For an intermediate size $\left(3 \mathrm{R}_{a c c} / 16\right)$ the oscillation was very weak and quasi-periodic.

(4) Essentially all the unstable flows were acompanied by the shedding of pairs of vortices.

(5) The instability always started by radial, "pumping" modes, which appeared as pulses in the density and velocity field along the accretion axis.

Analytical studies of Bondi-Hoyle flows treated the stability of two entities: the "accretion line" (on which matter form both sides collides) and the "shock cone" (which results from the supersoniic flow).

Analyses of the stability of the accretion line (which treated the linear regime and neglected pressure) demonstrated that (Cowie 1977, Soker 1990):

i. the accretion line is unstable against radial modes (both in 2D and in 3D).

ii. In $2 \mathrm{D}$, the accretion line is also unstable to tangential modes.

A simplified analysis of the stability of the shock-cone showed that (Livio et al 1991):

i. The shock cone is unstable to transverse deflections when the shock opening angle is large.

ii. For shock opening angles which are not extremely large, the flow is stabilized when the size of the accreting object exceeds some critical value.

iii. The instability always starts near the accreting object. 

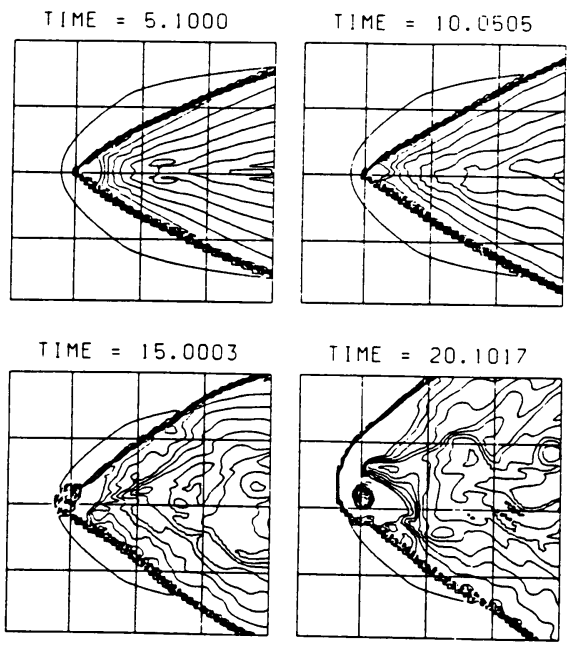

〈DENSITY>

Figure 1: Density contours as a function of time in a 2D run, $\gamma=5 / 3$, the Mach number is 3. Time is in units of $\mathrm{R}_{a c c} / \mathrm{C}_{s}$ (see text). From Matsuda et al. (1991b).

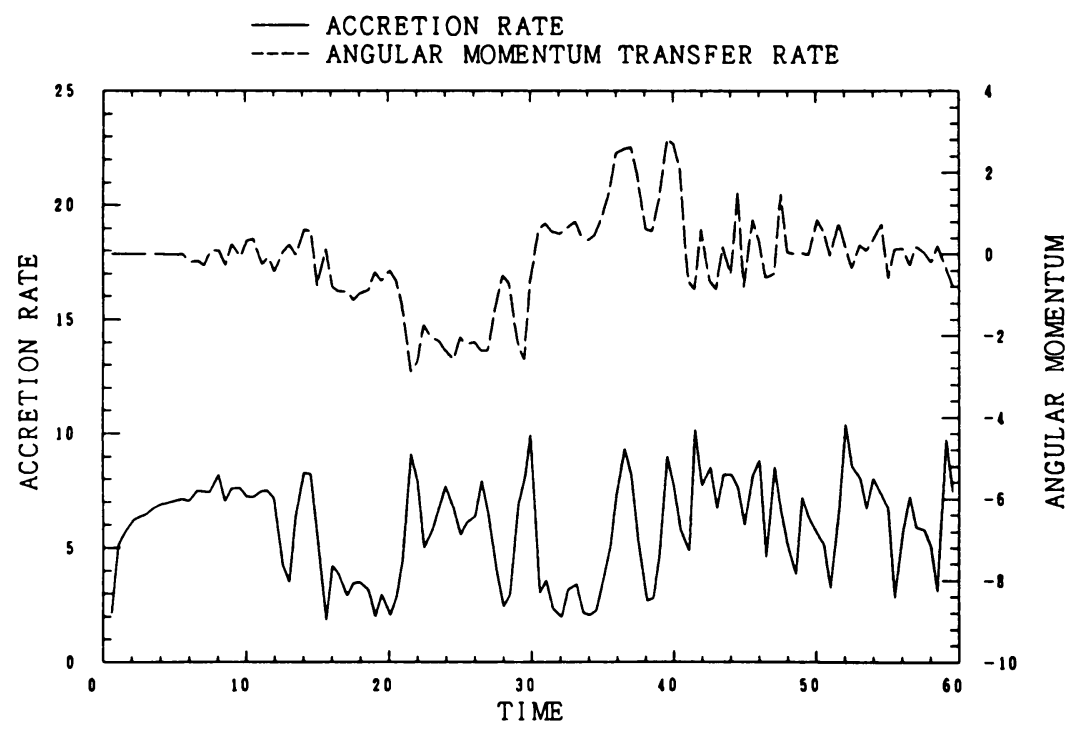

Figure 2: The mass and angular momentum accretion rates as a function of time for the same parameters as in Fig. 1. From Matsuda et al. (1991b). 

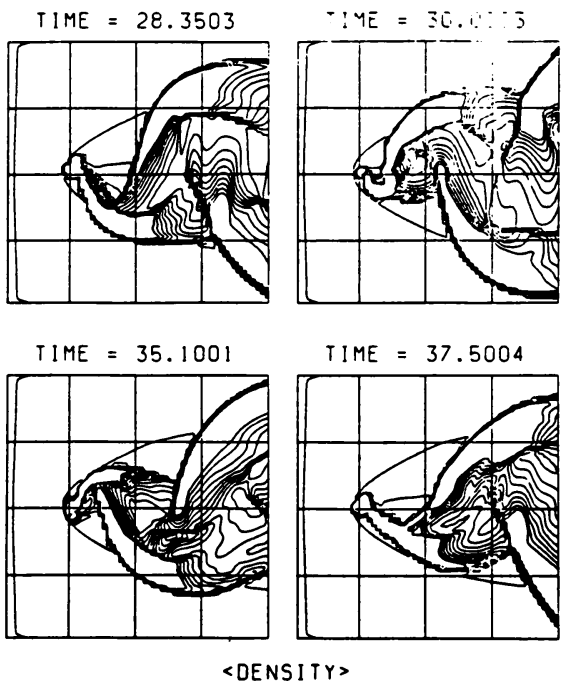

Figure 3: Density contours as a function of time in a 2D run, $\gamma=1.005$, the Mach number is 3. From Matsuda et al. (1991b).

ACCRETION RATE

--- ANGULAR MOMENTUM TRANSFER RATE

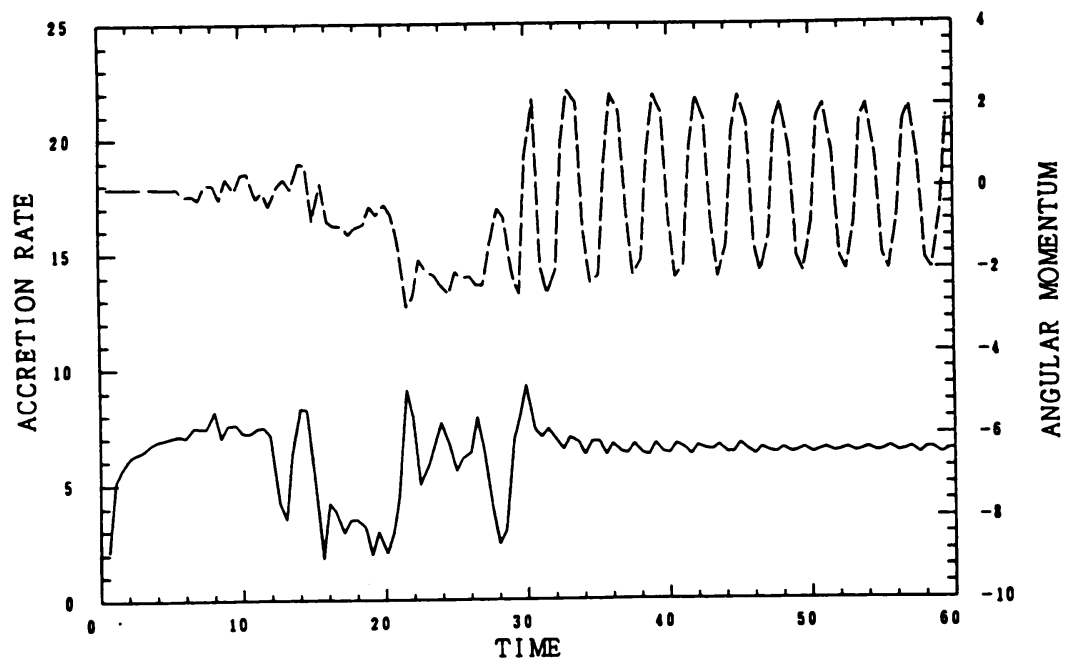

Figure 4: The mass and angular momentum accretion rates as a function of time. The parameters are the same as in Fig. 2 until $t=30$, then the size of the accreting object was increased from $R_{a c c} / 16$ to $5 R_{a c c} / 16$. From Matsuda et al. (1991b). 
The picture of the nature of the instability in 2D that seems to emerge from all of these studies is the following:

1. Radial modes of the accretion line instability are excited first (with the possible exception of cases in which the accreting object is very large).

2. The radial modes generate a "pumping-type" accretion process from the down-stream side, by causing large variations in the density and velocity along the accretion line.

3. The subsequent evolution depends on whether the shock-cone becomes unstable or not. The following possibilities seem to emerge:

(a) The shock-cone remains relatively narrow. This happens for low values of $\gamma(\gamma \sim$ 1) or when the accreting object is sufficiently large, so as to be able to accrete directly the mass inflow pulses which result from the radial modes. In this case the shock-cone remains stable and the flow is dominated by long wavelength tangential modes, which develop into a smooth, quasi-periodic oscillation.

(b) The shock-cone opening angel widens. This happens for large values of $\gamma(\gamma \sim 5 / 3)$ and for small sizes of the accreting object. The cause for the widening can either be the formation of a quasi-rigid atmosphere which forces material upstream, or the accretion of some angular momentum (which results in some type of circular motion). In this case, the shock-cone becomes unstable and a violent flip-flop oscillation ensues in the non-linear regime.

In addition, we note the formation of pairs of vortices behind the accreting object, in a somewhat similar manner to Karman vortex shedding in 2D incompressible flows past a circular cylinder.

In order to answer the second question posed at the beginning of this section, namely, does the instability appear also in 3D? Matsuda et al (1991b) performed a few preliminary calculations in $3 \mathrm{D}$. Their results can be summarized as follows:

1. The formation of a bow shock which is detached from the accreting object was observed (unlike the $2 \mathrm{D}$ case in which the shock was attached to the body).

2. Radial "pumping" modes developed.

3. Vortex rings-type structures were formed.

4. Distortions did appear in the shock-cone, accompanied by some side to side motion (Fig. 5), but no violent flip-flop of the entire shock was observed.

Our preliminary results seem to indicate that the flip-flop instability takes a less violent form in 3D (see also Boffin, this volume).

However, it should be remembered that in addition to some real differences in the physics (e.g. the absence of tangential modes in 3D and the fact that the 3D shock is more robust against deflections), the resolution of the calculations in $3 \mathrm{D}$ is necessarily lower than in $2 \mathrm{D}$. This results in a higher numerical viscosity and a larger damping of potential instabilities. Thus, at present, we cannot determine with certainty the nature of the flow in 3D. 

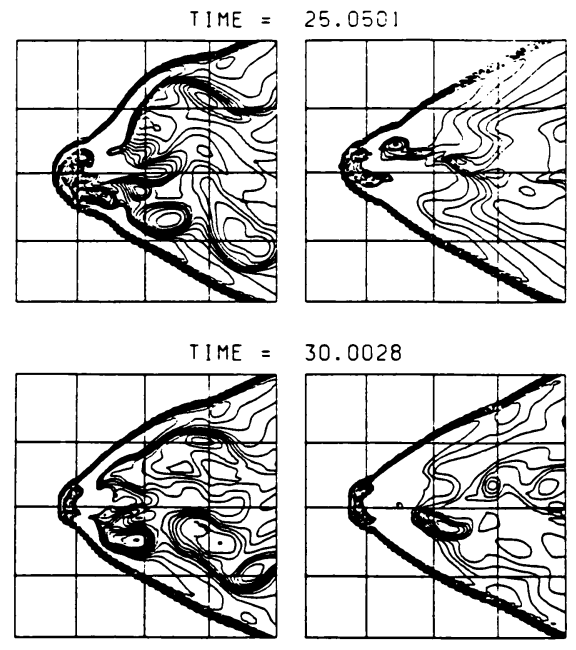

<DENSITY>

Figure 5: Density contours in a 3D run, $\gamma=5 / 3$, the Mach number is 3. Cuts in the $x-y$ (left) and x-z (right) planes are shown. From Matsuda et al. (1991b).

\section{Possible Observational Implications}

Assuming that the flip-flop instability indeed appears in real flows, it has some extremely interesting observational implicatoins for systems in which compact objects accrete from stellar winds and in particular for massive X-ray binaries. I shall now discuss some of these implications:

\subsection{Variability in the $X$-ray Luminosity}

During the oscillations of the shock-cone, the compact object (a neutron star or a black hole in the case of massive $X$-rav binaries) accretes material with a relatively high specific angular momentum of opposite signs, as the shock flip-flops from side to side. This leads to the following phenomenon: a temporary accretion disk froms, and as it starts to be fed by angular momentum of the opposite sign, the disk collapses onto the compact object. This is followed by the formation of a disk rotating in the opposite sense. The sudden accretion events should lead to flares in the $X$-ray luminosity. Thus, the prediction is that in wind-fed sources, the X-ray luminosity should exhibit irregular bursts on a timescale of minutes to hours (a few time $R_{a c c} / V$, where $V$ is a typical wind velocity). An examination of the observational situation reveals that indeed sources that are believed to be wind-fed, such as 4U1700-37, Vela X-1 and GX301-2 show such irregular X-ray bursts (e.g. Doll and Brinkmann 1987, White 198.5, Watson, Warwick and Corbett 1982).

Another source that showed quasi-regular bursts during a major outburst, that were interpreted as resulting from the flip-flop instability, is EXO $2030+375$ (Parmal et al 1989, Taam, Fryxell and Brown 1988). However, it should be remembered that this system contains a Be star (see van den Heuvel and Rappaport 1987, for a review of such systems) and 
Be stars are known to exhibit variability which may be related to pulsations or magnetic activity (e.g. Balona 1990, Baade, this volume). It is therefore not entirely clear whether flip-flop oscillations are the real cause for the behaviour of EXO $2030+375$.

\subsection{The Spin History}

Neutron stars accreting from the winds of early type companions should experience a torque with alternating signs during the disk formation episodes. Therefore, an important implication of the flip-flop instability is that the spin history of the neutron star should be characterized by shot-noise type spin-up and spin-down episodes (neglecting other effects such as crust-interior coupling).

Interestingly enough, most if not all of the wind-fed sources show indeed an alternating spin-up, spin-down behaviour, while the neutron stars in systems that are fed by Roche lobe overflow show a relatively smooth spin-up history (Fig. 6). It is in fact possible to determine which systems are wind-fed simply from an examination of their spin histories (compare for example in Fig. 6, Vela X-1 and GX 301-2 which are wind-fed to SMC X-1 and Her X-1 which are fed by Roche lobe overflow). In particular, the angular frequency of Vela X-1 shows variations at a rate of $|\dot{\Omega} / \Omega|=(5.8 \pm 1.4) \times 10^{-3} \mathrm{yr}^{-1}$, with the sign of the derivative reversing on timescales as short as the resolution of the data (Deeter et al 1989).

\section{Conclusions and Remaining Problems}

Based on the numerical and analytical work described in the previous sections we note the following conclusions:

1. In two-dimensions, Bondi-Hoyle accretion flows are non-steady. They exhibit a "flipflop" instability even in the homogeneous case.

2. More high-resolution calculations are needed in order to determine the existence and nature of the instability in three-dimensions.

3. The instability (if it indeed occurs in realistic flows) can have important implications for the possibility to form accretion disks in wind-fed sources and for the spin history of the neutron stars in massive $\mathrm{X}$-ray binaries. In particular, temporary disks of an alternating sense of rotation should form.

4. Observations of massive $X$-ray binaries are consistent with the predictions based on the existence of the flip-flop instability. In particular, these sources show: (i) irregular flares in their X-ray luminosity and (ii) a random walk-type spin-up, spin-down behaviour.

5. An interesting question is related to Cyg X-1. The compact object in this system (a black hole candidate) is thought to be a wind accretor (e.g. White 1985). Yet, its $\mathrm{X}$-ray light curve is quite smooth (e.g. Mason et al 1974) and the system exhibits some signatures of a relatively cool accretion disk (Done et al 1990). The question is: why doesn't the system show the same intensity variations as the other wind-fed sources? This leads to the following interesting possibilities: (1) either the black hole is in fact fed by Roche lob overflow, or (2) the flip-flop instability is not operative in this case. The second possibility is particularly intriguing. I note that it is much easier for an accretion disk to form around a black hole than around a magnetized neutron star, because the innermost stable orbit is much smaller in radius than a typical magnetospheric radius. If a relatively stable disk forms around the black hole 
and spreads due to viscoity, its presence may suppress the development of the flip-flop instability (by effectively increasing the size of the accreting object). If this explanation is correct (which is not clear at all presently), this suggests the exciting possibility that the presence of black holes (rather than magnetized neutron stars) in massive X-ray binaries can be detected simply via the absence of the intensity variations associated with the flip-flop instability.

Acknowledgement: Most of the work presented here was done in collaboration with Takuya Matsuda, Uli Anzer and Noam Soker. This work has been supported in part by the Director's Research Fund at the Space Telescope Science Institute.

\section{References}

Balona, L.A. (1990) M.N.R.A.S., 245, 273.

Blondin, J.M., Kallman, T.R., Fryxell, B.A. and Taam, R.E. (1990), Ap.J., 356, 591.

Bondi, H. and Hoyle, F. (1944) M.N.R.A.S., 104, 273.

Cowie, L.L. (1977) M.N.R.A.S., 180, 491.

Davies, R.E. and Pringle, J.E. (1980) M.N.R.A.S., 191, 599.

Deeter, J.E., Boynton, P.E., Lamb, F.K. and Zylstra, G. (1989) Ap.J., 336, 376.

Doll, H. and Brinkmann, W. (198i) Astron. Ap., 173, 86.

Done, C. Mushotzky, R., Mulchaey, J. and Armand, K.A. (1991) paper present at 'Structureand Emission Properties of Accretion Disks', IAU Colloq. 129, Paris.

Fryxell, B.A. and Taam, R.E. (1988) Ap.J., 335, 862.

van den Heuvel, E.P.J. and Rappaport, S. (1987) in Physics of Be Stars, A. Slettebak and T.D. Snow (ed.), Cambridge University Press, Cambridge, p. 291.

Lamb, D.A. (1988) in Phsyics of Neutron Stars and Black Holes, Y. Tanakka (eds.), Universal Academy Press, Tokyo, p. 21.

Livio, M. (1988), in 'The Symbiotic phenomenon', eds. J. Mikolajewska et al. (Dordrecht: Kluwer Academic Publishers), p. 149.

Livio, M. (1991) in 'Structure and Emission Proporties of Accretion Disks', IAU Colloq. 129, eds. C. Bertout et al. (Gif Sur Yvette: Editors Frontieres) p. 263.

Mason, K.D., Hawkins, F. J.. Sanford, P.W., Murdin, P. and Savage, A. (1974) Ap.J., 192, L65.

Matsuda, T., Inoue, M. and Sawada, K. (1987) M.N.R.A.S., 226, 785. 
Matsuda, T., Sekino, N., Sawada, K., Shima, E., Livio, M., Anzer, V. and Börner, G. (1991a), Astron. Ap., in press.

Matsuda, T. Ishii, T., Sekino, N. Sawada, K., Shima, E., Livio, M. and Anzer, V. (1991b) M.N.R.A.S., in press.

Nagase, F. (1989) Pub. Astron. Soc. Japan, 41, 1.

Parmar, A.N., White, N.E., Stella, L., Izzo, C. and Ferri, P. (1989) Ap. J., 338, 359.

Sawada, K., Matsuda, T., Anzer, V., Börner, G. and Livio, M. (1989) Astron. Ap., 231, 263.

Soker, N. (1990) Ap. J., 358, 545.

Soker, N. (1991) Ap. J., in press.

Taam, R.E. and Fryxell, B.A. (1988) Ap.J., 327, L73.

Taam, R.E., Fryxell, B.A. and Brown, D.A. (1988), Ap.J., 331, L117.

Taam, R.E., Fu, A. and Fryxell, B.A. (1991) Ap.J., in press.

Watson, M.G., Warwick, R.S. and Corbett, R.H.D. (1982) M.N.R.A.S, 199, 915.

White, N.E. (1985) in Interacting Binaries, eds. P.P. Eggleton and J.E. Pringle, (Dordrecht: Reidel), p. 249. 

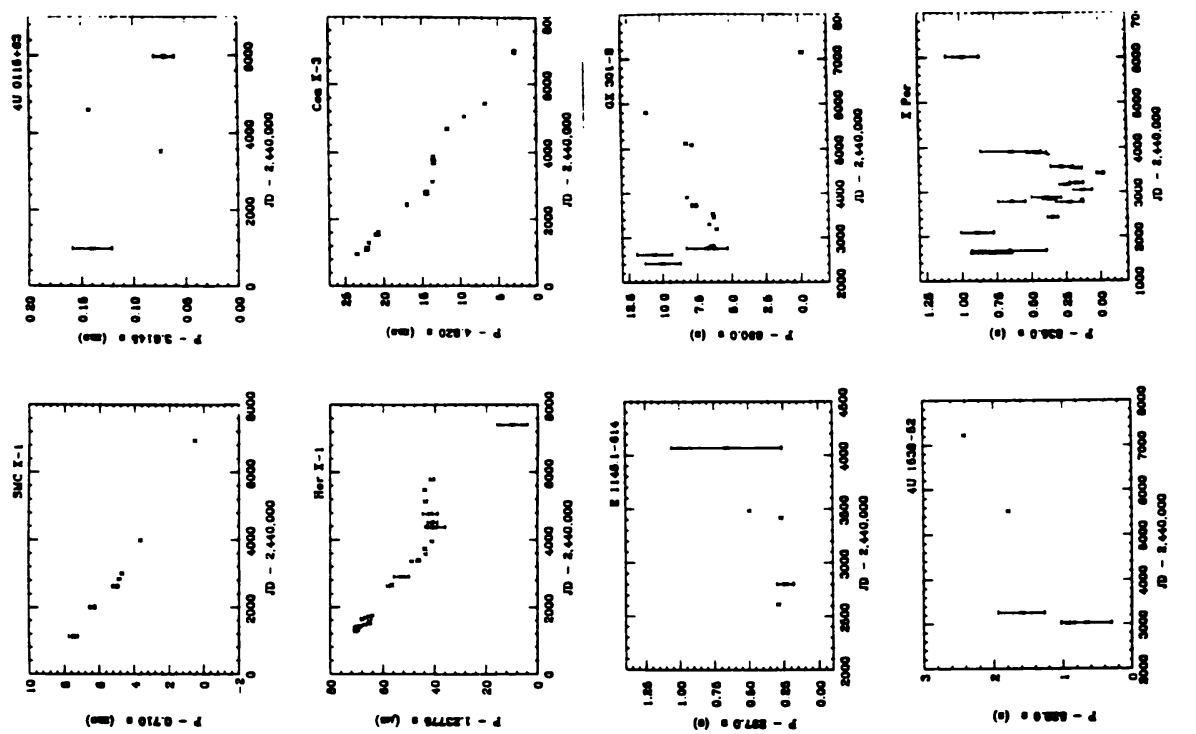

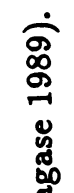
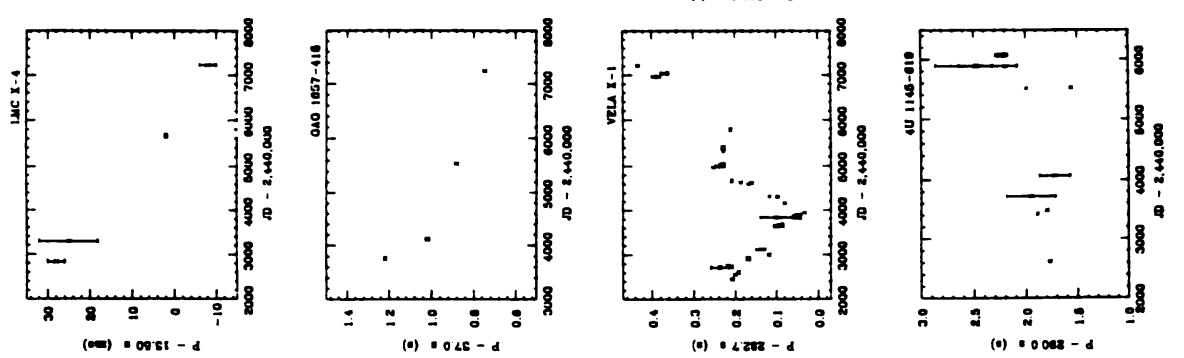

Z
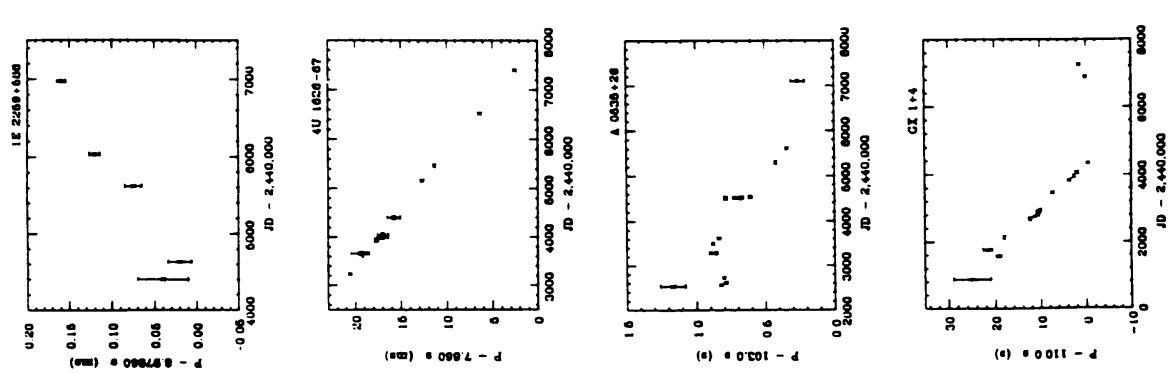